\title{
Perspectives of Water Level Measurement in Plastic Pipes Using Wideband Horn Antenna
}

\author{
Zahid Farid \\ Department of Electrical Engineering, \\ University of Science and Technology, \\ Bannu, Pakistan \\ zahidfarid@ustb.edu.pk
}

\author{
Zeeshan Najam \\ The Ultimate Engineering \\ Enterprises Ltd, \\ Islamabad, Pakistan \\ shaani75@hotmail.com
}

\author{
M. Y. Ali Khan \\ Department of Electrical Engineering, \\ Gomal University D.I.Khan, \\ Dera Ismail Khan, Pakistan \\ myousafak@gu.edu.pk
}

\author{
Sheeraz Ahmed \\ Department of Computer Science, \\ Iqra National University, \\ Peshawar, Pakistan \\ sheerazahmed306@gmail.com
}

\author{
Sarah Akhtar \\ Department of Physics, \\ Federal Urdu University of Arts Sciences \& Technology, \\ Karachi, Pakistan \\ sara@fuuast.edu.pk
}

\begin{abstract}
This paper is concerned with the investigation of a possible working principle of a microwave sensor for the measurement of water levels in plastic pipes. According to the research, most current sensors for water level measurement require an unobstructed path from the sensor in the water and therefore, the integrity of the pipes, in which water flows, will be compromised. The proposed sensor will work at microwave frequencies to measure the flow in the pipes from the outside. This would be non-invasive and non-intrusive. A beam of microwave energy from an antenna is directed towards a water surface within a pipe and the reflection due to the surface of the water will be isolated and knowledge of the pipe dimensions and properties will allow the depth of water to be calculated. Knowledge of the pipe drop may also allow flow to be estimated. It is observed that at microwave frequencies the reflection from the surface of the water is many times greater than the reflection from the surface of a typical plastic pipe.
\end{abstract}

Keywords-PVC pipe; CST; antenna and return loss

\section{INTRODUCTION}

Wter usage of water is commonly monitored by water meter installation. If there are no meters, users are billed a constant amount of charge irrespective of the amount of water they consume. This situation causes not only a deficit to the country's revenue of the recourse but also extra charges in some cases at the consumer level. So, there is a need of a monitoring system which could give us continuous information about the water usage at any level. With the advancement of electronic measurement and control systems, this problem can be solved by providing a microwave sensor which could be installed on PVC pipes having continuous water flow. Pulse, phase difference and Doppler methods along with pressure sensing are four popular ways of electronic distance measuring [1]. These all offer possible situations for the water sensor. Measuring the time between transmission and reception of a pulse transmitted by a transmitter, the distance can be calculated as:

$$
2 d=c \Delta t^{\prime}=c\left(t_{R}-t_{E}\right)
$$

where $d$ is the distance between instrument and target, $c$ is the light velocity in the medium, $t_{R}$ is the pulse flight time, $t_{E}$ is the time of pulse departure. It can be seen that the accuracy of the distance measurements depends on the accuracy of flight time measurement. An accuracy of $0.1 \mathrm{nsec}$ is equivalent to an accuracy of $15 \mathrm{~mm}$. For example a $150 \mathrm{~mm}$ diameter pipe would require a 2 nsec delay before a pulse reflected from the bottom side of a pipe should be seen. For the transmitter, the narrower the impulse transmitted the better the resolution of the target that will be achieved. However, narrow pulses in time tend to larger bandwidths in frequency. Therefore, pulse method distance measurement systems are required to be broadband. It turns out that the broader the bandwidth of the measuring device, the better the resolution in the accuracy of the measurement.

In the phase difference method, distances are measured by measuring the phase difference of continuous waves. Light waves and radio waves are used as carrier waves in this technique. A modulated signal on the carrier wave is transmitted from the transmitter towards the reflector and back to the electronic distance measurement (EDM) device, where it is picked up by the receiver. It is at the receiver where the phases of transmitted and received signals are compared and phase lead is calculated. Phase comparison only gives us the lead time. A time equivalent to the number of full cycles elapsed during the flight of a specific signal has to be added to obtain the total time of flight $\Delta t^{\prime}$.

$$
\Delta t^{\prime}=m . t^{*}+\Delta t
$$

where $m$ is the integral number of full wavelengths over the measuring path, $t^{*}$ is the elapsed time for one full cycle of the 
modulation signal. The phase difference method can be implemented in a narrow band form. The level of accuracy depends on the frequency of the carrier used and the level to which the phase difference between the transmitted and received waveforms can be resolved. The most popular technique for electronic distance measurement is a Doppler system consisting of a microwave transmitter and receiver. The principle behind the technique is that the instrument moves with a speed of $v$ towards the reflecting surface which is placed at a distance $d$ from the instrument. The surface of the target reflects the transmitted signal and is captured by the receiver. The emitted frequency is expressed as:

$$
f_{T}=v / \chi
$$

where $v$ is velocity of light and $\lambda$ is the wavelength of the emitted radiation. The frequency which arrives at the reflecting surface is:

$$
f_{s}=(c+v) / \chi
$$

where $v$ is the speed of the instrument, relative to the reflecting surface. At the receiver the received frequency is given by:

$$
f_{R}=(c+2 v) / \lambda
$$

The Doppler frequency $f_{D}$ can be achieved by subtracting $f_{T}$ from $f_{R}$ :

$$
f_{D}=f_{R}-f_{T}=(c+2 v) / \lambda-v / \lambda=2 v / \lambda
$$

The procedure for measuring the Doppler frequency depends on the type of waves used. For radio waves it is measured by counting the cycles of the Doppler signal per second. Equation (6) can be written as:

$$
v=1 / 2 f_{S} \lambda
$$

The distance travelled by the instrument between time $t_{l}$ and $t_{2}$ is:

$$
d_{12}=\int_{t 1}^{t 2} \frac{1}{2} f s \lambda
$$

Close inspection of (8) shows that for a stationary system this equation yields the fundamental wavelength which is equivalent to the phase difference method for distance measurement. This system is narrow band. Such a system could also be used to measure perturbations on the surface of water in a pipe.

Principle of microwave reflections has been used in tumor and breast cancer detection [2-5] in the medical field, and in analyzing rubber containing iron particles [6], mineral treatment processes [7], high accuracy microwave ranging system [8], electronic distance measurements [9] and most importantly cable fault detection $[10,11]$ in the industrial field. The proposed technique is to isolate the reflection coefficient, $S_{11}(\mathrm{~dB})$, of a wideband antenna held on the top of flowing water inside a PVC pipe. For this, it is necessary to study the behavior of the reflected signal from a planar normal surface and is best illustrated using the example of time domain step reflection. In Figure 1 we see an abstracted version of the measurement scenario. Transmitter and antenna have been replaced with an oscilloscope. The air way between the antenna and the pipe is represented by a section of transmission line and the pipe and water are represented as a device under test.

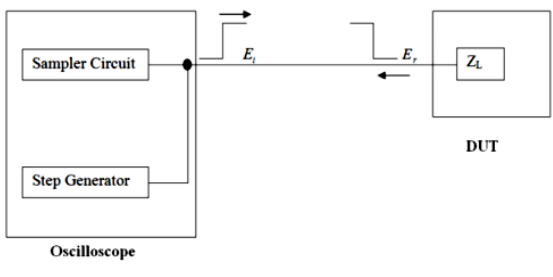

Fig. 1. A block diagram of a reflectometer

The step generator produces a positive-going incident wave that is applied to the device under test via a transmission line. The step travels along the transmission line at the velocity of propagation of the light. If the load impedance of DUT is equal to the characteristic impedance of the line, no wave is reflected and all that will be seen on the oscilloscope is the incident voltage step recorded as the wave passes the point on the line monitored by the oscilloscope as shown in Figure 2. If there is a mismatch at the DUT then a part of the incident wave will be reflected back and the wave form on the oscilloscope will embed some of the incident and reflect wave as shown in Figure 3 .

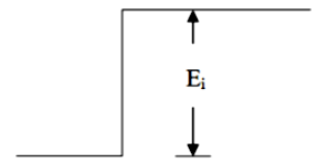

Fig. 2. Oscilloscope display of when there is no mismatch, $\mathrm{E}_{\mathrm{r}}=0$

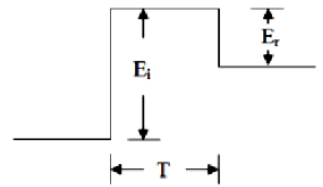

Fig. 3. Oscilloscope display when there is any mismatch, $\mathrm{E}_{\mathrm{r}} \neq 0$

The reflected wave is readily identified since it is separated in time from the incident wave. This time is also valuable in determining the length of the transmission system from the monitoring point to the mismatch. Letting $\mathrm{D}$ denote this length:

$$
D=v_{p} \times T / 2
$$

where $v_{p}$ is the propagation velocity and $T$ is is transit time from monitoring point to the mismatch and back again. One of the advantages of time domain reflection (TDR) is its ability to handle cases involving more than one discontinuities. If we look at the example in Figure 4, it can be seen that there are more than one impedance mismatches occurring in this system.

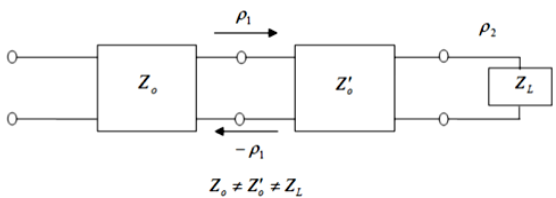

Fig. 4. Cable with more than one discontinuities 
$\rho_{1}, \rho_{2}$ are the reflection co-efficients given by:

$$
\begin{aligned}
& \rho_{1}=\frac{z_{0}^{\prime}-z_{0}}{z_{0}^{\prime}+z_{0}} \\
& \rho_{2}=\frac{z_{L}-z_{0}^{\prime}}{z_{L}+z_{0}^{\prime}}
\end{aligned}
$$

If we consider that $z_{0}^{\prime} \geq z_{0} \geq z_{L}$ then the display which will appear on the oscilloscope will be like the one shown in Figure 5. The important point here to note is that as we go further down the cable, the measurement accuracy goes down. In our case of measurements, for determining the height of water inside the pipe, the electromagnetic waves will propagate in air with a propagation velocity approximately equal to $3 \times 10^{8} \mathrm{~m} / \mathrm{sec}$. Figure 6 shows the transmission line equivalent circuit for the water measurement device.

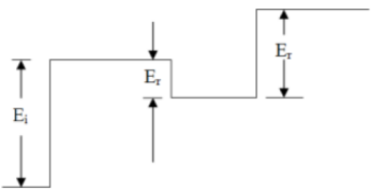

Fig. 5. Oscilloscope display for a cable with multiple discontinuities

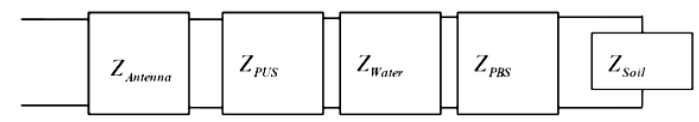

Fig. 6. Transmission line equivalent circuit for the water measurement device

Here $z$ stands for impedance, PUS stands for pipe upper surface and $P B S$ stands for pipe bottom surface. In the model we can assume that the impedances in order of magnitude are likely to be $z_{\text {Water }}$, $z_{\text {Antenna }}$ (including connectors), $z_{\text {Soil }}$, zPipesurface. A layer model with approximate thicknesses and dielectric properties of pipe, water, soil and air is shown in Table I. Note that it is assumed that there are no magnetic materials in the system. In this system, water would have the largest reflection followed by soil. Note the soil below the pipe could be treated with iron or salt to increase its reflection/mismatch thereby providing a calibration surface for the system. However, any conductive surface below the pipe may increase the number and magnitude of echoes into the system.

TABLE I. LAYER MODEL

\begin{tabular}{|c|c|c|}
\hline Impedance & & Permittivity \\
\hline $0 \leq \mathrm{Z} \leq 1 \mathrm{~m}$ & Air & 1 \\
\hline $5 \times 10^{-3}$ & PVC & $\approx 2-4$ \\
\hline $0 \leq \mathrm{Z} \leq 150^{-3} \mathrm{~m}$ & Air2 & 1 \\
\hline $150 \times 10^{-3} \mathrm{~m}-$ Air2 & Water & $\approx 55-90$ \\
\hline $5 \times 10^{-3} \mathrm{~m}$ & PVC & $\approx 2-4$ \\
\hline $0 \leq \mathrm{Z} \leq-\infty$ & Soil & $\approx 10-40$ \\
\hline
\end{tabular}

\section{SIMULATIONS AND EXPERIMENTAL RESULTS}

\section{A. Distance Measurement Inferred from Reflections from the} Metal Surface.

The purpose of these experiments is to determine the relative level of reflected energy that could be available to a time of flight measurement system from the normal surface of water within a PVC pipe. In the system a transducer emits a wave towards the pipe. At the surface of the pipe the wave is partially reflected, partially absorbed and partially transmitted. This happens again at the boundary between the pipe material and the air in the pipe, again at the surface between the air in the pipe and any water in the pipe, again at the boundary between the water and the lower inner surface of the pipe and finally at the boundary between the PVC and the soil under the pipe. According to [10] and the properties of air, PVC, water and soil at microwave frequencies, the air-water boundary will reflect the largest proportion of energy whilst transmitting the least. The pipe wall is planar along its long axis and curved at right angles to its long axis. However, for the initial measurements the pipe wall can be represented by a planar sheet of PVC with suitable thickness. If the sheet is large when compared to the wavelength, unwanted scattering from the non normal axis of the pipe and diffractions from the edges of either the pipe or the planar sheet will be avoided.

At first, the system consisting of a measurement unit, an antenna and a pipe with water is represented by a sheet of polyvinyl chloride (PVC) of large extent above a copper sheet. The copper sheet represents an ideal reflector and the PVC sheet the top surface of the pipe. Large planer surfaces also allow avoidance of problems with orientation and non perpendicular reflections. The system was first modeled in CST. In the model a plane wave was used for excitation. This scenario used an impulse and is therefore a representative of the pulse distance measurement system. Two plates of 250mmx250mmx3mm (PVC and copper) were spaced $1 \mathrm{~m}$ from one another and excited by plane wave $0.2 \mathrm{~m}$ away and an output point was set at a distance of $0.8 \mathrm{~m}$ away from the PVC plate. This experiment is shown in figure 7.

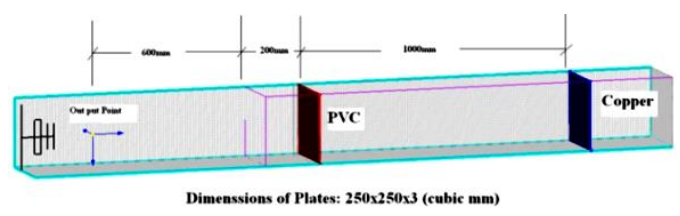

Fig. 7. Plane wave illuminations of PVC and copper plates with $1 \mathrm{~m}$ distance

The results after simulation are shown in Figure 8 . According to the results the first reflection is from the PVC plate which occurred at 3.202 nsec. Here it should be explained that this time is the total time from excitation point to the surface of PVC plate and then back to the output point shown in Figure 8. This time equates to a distance of $0.961 \mathrm{~m}$. The measured distance is $0.985 \mathrm{~m}$, therefore there is a $2.43 \%$ error. Similarly for the copper plate, the reflection occurred at $9.912 \mathrm{nsec}$ which equates to a distance of $2.974 \mathrm{~m}$. The actual distance was $2.997 \mathrm{~m}$ having a $2.3 \%$ error. One point here to be noted is that the speed of light in all these measurements was chosen to be $3 \times 10^{8} \mathrm{~m} / \mathrm{sec}$. The errors in the measurement can be reduced by taking the actual speed of light, which is $2.998 \times 10^{8} \mathrm{~m} / \mathrm{sec}$. This experiment shows that a sensor would be possible and indicates that a return from a copper plate has approximately 12 times the magnitude of a return from the 
PVC sheet. The experiment also shows that returns $1 \mathrm{~m}$ apart lead to a time difference of approximately 8 nsec. From this we can assume that for a $150 \mathrm{~mm}$ diameter pipe, the time difference between the returns is likely to be: $8 \times 10^{-9}[1 / 0.15]=1.2 \mathrm{nsec}$. This means that if the receiver started 2 nsec before the first pulse arrived and run for $4 \mathrm{nsec}$ all the required data would be captured.

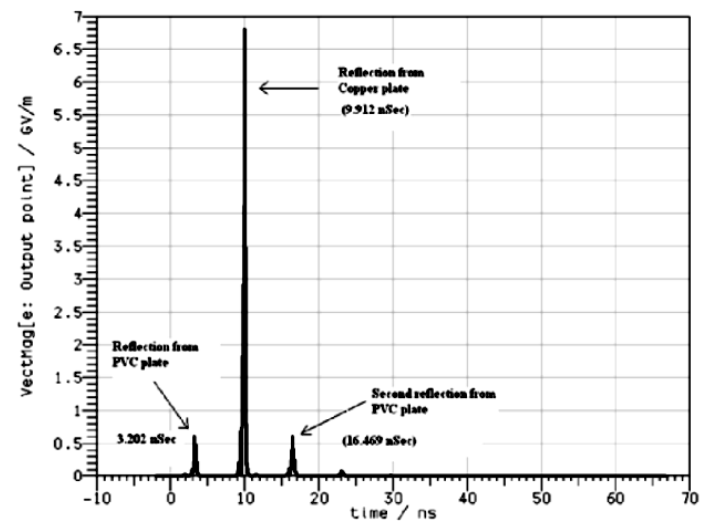

Fig. 8. The time domain representation of the reflections from a copper plate behind a PVC sheet.

Water was added in the next simulation (Figure 9). Note that whilst we can reasonably assume materials and conditions of the pipe to be constant, we cannot assume the conditions of materials of the surface below the pipe. One way to create repeatable conditions would be to cover the bottom face of the pipe with a good conductor such as copper foil. The next simulation therefore contains a conductive copper plate beyond the water. The results are shown in Figure 10.

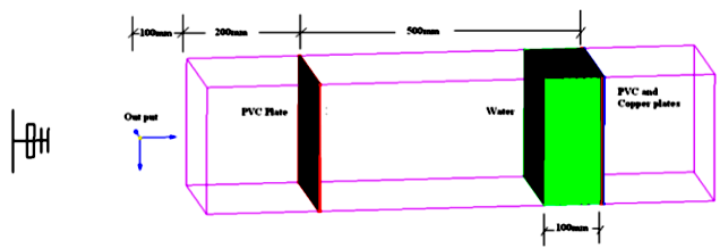

Fig. 9. Water and air between two PVC sheets terminated by a copper plate short circuit

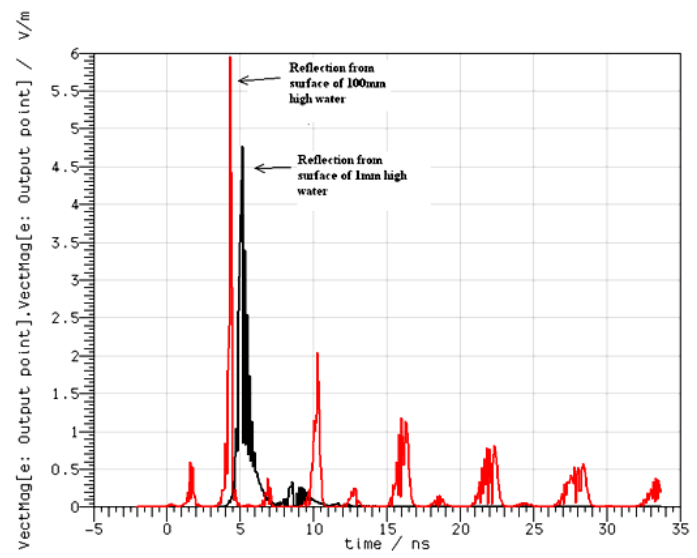

Fig. 10. The time domain response of several levels of water between two PVC plates truncated by a copper plate
In this case the gate of the experiment was reduced from $1 \mathrm{~m}$ to $0.5 \mathrm{~m}$. It can be seen that the first return is the top of the pipe and its amplitude does not change for no water, $100 \mathrm{~mm}$ of water and $1 \mathrm{~mm}$ of water. This reassures that this is a single reflection rather than several reflections merged together. The water surface produces good returns at both $100 \mathrm{~mm}$ and $1 \mathrm{~mm}$. The return at $100 \mathrm{~mm}$ water is distinct from the metal plate. The return at $1 \mathrm{~mm}$ is not distinct from the metal plate. In Figure 11 the return from metal plate is constant in time. These results also show multiple reflections in time as energy is reflected to and from each boundary in the system. The periodicity of these reflections indicates that the plates are large enough to avoid significant diffractions at their edges and that the copper plate terminates the gate effectively. One important point here to note is that if copper plate was not backing the whole structure we would have a minute reflection for from the end PVC plate. For this purpose a small amendment was made with the structure in Figure 10 by eliminating the copper plate from behind so that the whole of the transmitted wave is absorbed and not reflected. The results for this case are shown in Figure 11 . In this case a $1 \mathrm{~mm}$ height water did not give any reflection but the reflections from the surface of $100 \mathrm{~mm}$ height water and front end PVC plate remained fixed in time.

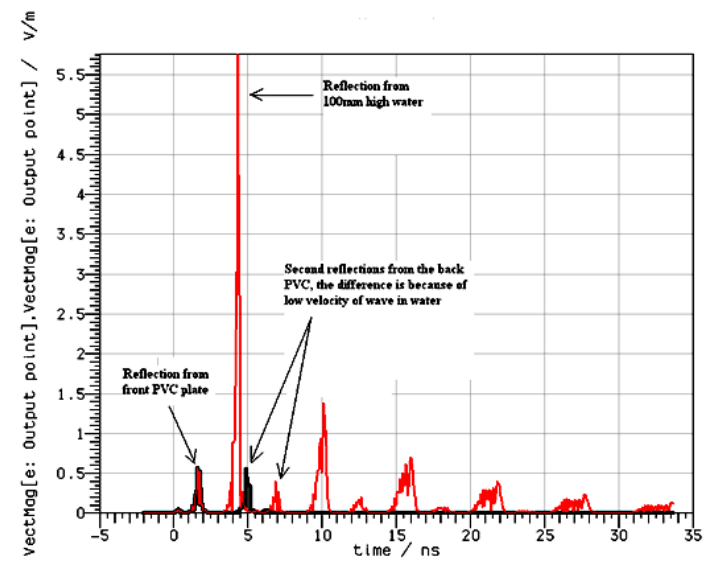

Fig. 11. Time domain representation of reflections from different levels of water between PVC plates

A $1 \mathrm{~m}$ long with $0.2 \mathrm{~m}$ radius $\mathrm{PVC}$ pipe, having different levels of water was also simulated in CST micro stripes (Figure 12). A plane wave excitation was given from the top. The results for no water and almost half full water pipe are shown in Figure 13. Simulations suggest that in general the returns from the pipe are less than those from planar experiments. This is most likely due to the scattering caused by the curved surface of the pipe. This may mean that the polarization of the incident wave may be used to maximize the return or minimize the scattering or both. The two traces in Figure 14 show an empty pipe (red trace) and a pipe that is half full (black trace). The red trace shows only reflections from the upper and lower surfaces of the pipe. The black trace shows the reflection from the upper surface of the pipe and a significant return from the surface of the water in the pipe. All the responses are within the time frame of 4 nsec. Copper sheet absence (conductive foil at the lower outer of the pipe) significantly reduced reflections and therefore cleared up the trace for peak detection. 


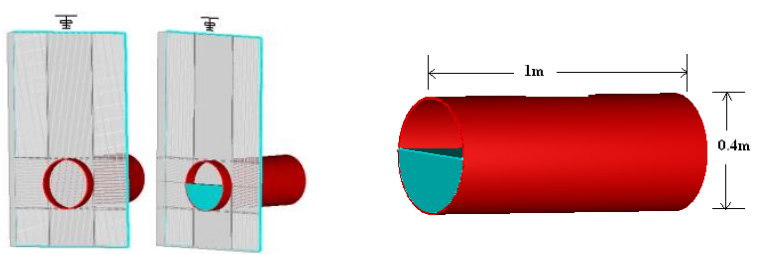

Fig. 12. Simulation of $1 \mathrm{~m}$ long PVC pipe having different levels of water

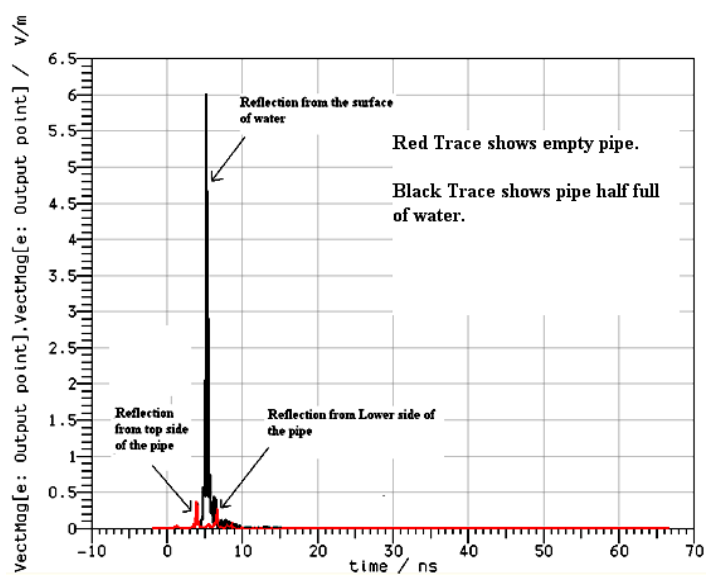

Fig. 13. Simulation results for $1 \mathrm{~m}$ PVC pipe with different levels of water

\section{B. Experimental Results for Static Water in a Tank}

The aim of this experiment was to measure the reflections from the surface of water only. The experiment is shown in Figure 14.

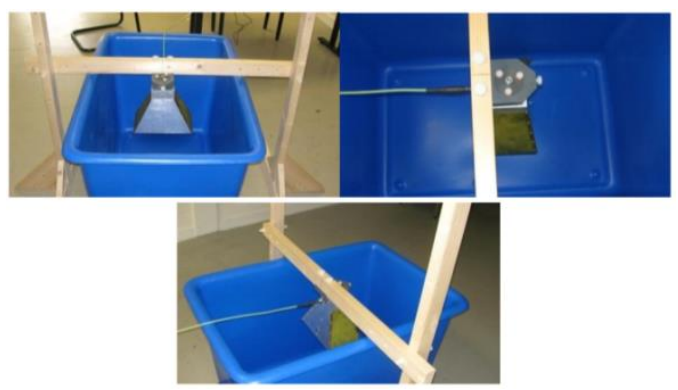

Fig. 14. Layout of the experimental rig for measuring reflections from the surface of static water

The reflection coefficient of the horn antenna was measured for the empty tank and tank having different levels of water (16mm, 32mm and 48mm). A large container was used in order to avoid any reflections from the edges. In front of the horn no metallic objects were allowed, however there may have been metal in the floor. The instrument used was Rhodes and Schwarz R\&S ZVL13 [11], which was able to produce results in time domain. The instrument's bandwidth was $9 \mathrm{KHz}$ to 13.6GHz. The results are shown in Figure 15 which shows that the peaks for the four scenarios are distinct.

There is a great deal of noise in the results indicating a metal plate or bars in the floor below the experiment. The dynamic range of the measurements is $\approx 10 \mathrm{~dB}$. The range of the measurements is less than $2 \mathrm{nsec}$.

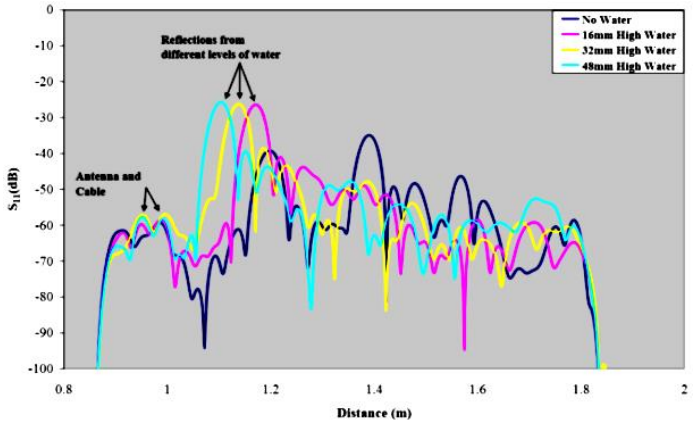

Fig. 15. Reflections of water levels inside a plastic tank

\section{Experimental Results for Static Water inside a Pipe}

A PVC pipe having an external diameter of $11 \mathrm{~cm}$ was used with and without water. At first some water inside the pipe was made static by closing both sides of the pipe. The reflection coefficient was again measured for the empty pipe and then for the pipe having some water inside it. The setup is shown in Figure 16. At first the challenge was to find out the reflection of electromagnetic waves coming from the upper and lower ends of the pipe. For this, a sheet of copper foil was selected to be put on the lower and upper ends of the pipe. Measurements were performed for a frequency span of $9 \mathrm{KHz}$ to $13.6 \mathrm{GHz}$ for the empty pipe. The purpose of these measurements was to find the reflections coming from the lower and upper surfaces of the pipe. Results are given in Figure 17. Once the reflections from the upper and lower end of the pipe were found, another set of measurements was performed to find out the effects of water inside the pipe and the results are shown in Figures 18 and 19. In these figures, front means front end of the PVC pipe and bottom means bottom end of the same pipe. The difference in the curves of Figures 18 and 19 is caused by the presence of a metal plate beneath the pipe during the measurements of Figure 19.

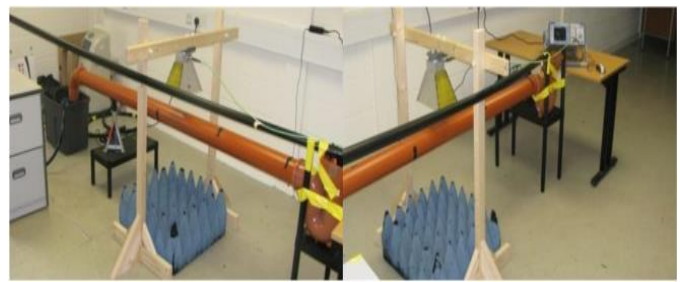

Fig. 16. Layout of the experimental rig for water to flow inside the pipe

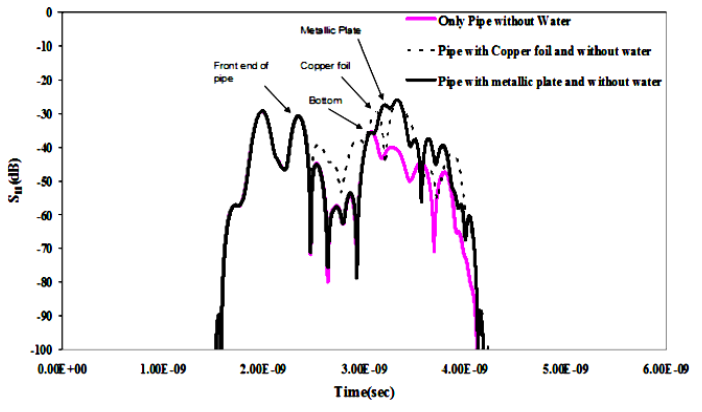

Fig. 17. Reflections of the upper and lower surfaces of a pipe and from a metallic surface placed beneath the pipe 


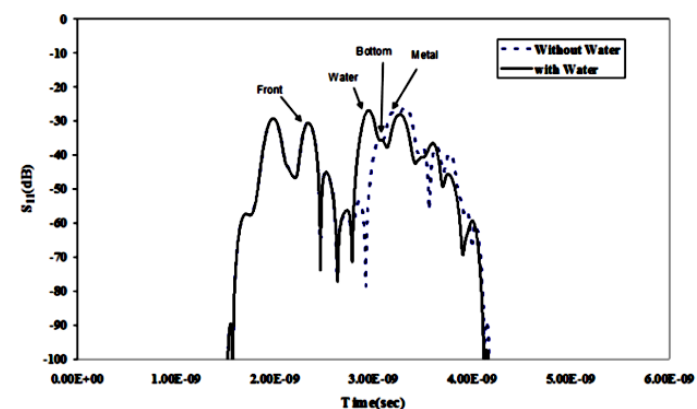

Fig. 18. Reflections from the surface of water inside a pipe with a copper plate beneath

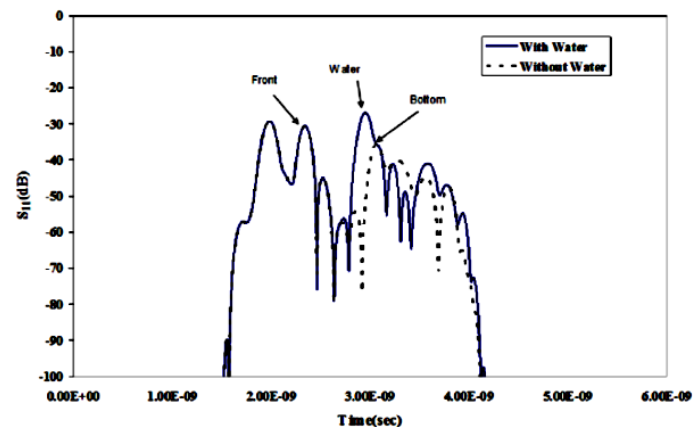

Fig. 19. Reflections from the surface of water inside a pipe

\section{Experimental Results for Flowing Water with Different Heights Inside the Sewerage Pipe}

The aim of these experiments was to measure the reflections of flowing water inside a pipe. To allow water flow, the pipe was arranged with a 2 degree slope. This arrangement means that the antenna was slightly off the perpendicular. However, an initial test (not shown here) suggests that the degree of movement of the main beam did not have a significant effect. For these experiments, some arrangements were made to achieve variable and smooth flows. A submersible water pump was placed inside a water reservoir which could push water through a small pipe (black) to the start of the main pipe (brown). A valve separate from the motor was used in order to have two different water levels in the main pipe. These levels were approximately $9 \mathrm{~mm}$ and $21 \mathrm{~mm}$ high. The results for these flowing water levels inside the pipe are shown in Figures 20 and 21. In these figures "with water" (a)(g) represent seven different measurements for flowing water at $9 \mathrm{~mm}$ and $21 \mathrm{~mm}$ heights inside the PVC pipe, to check the repeatability of the measurements.

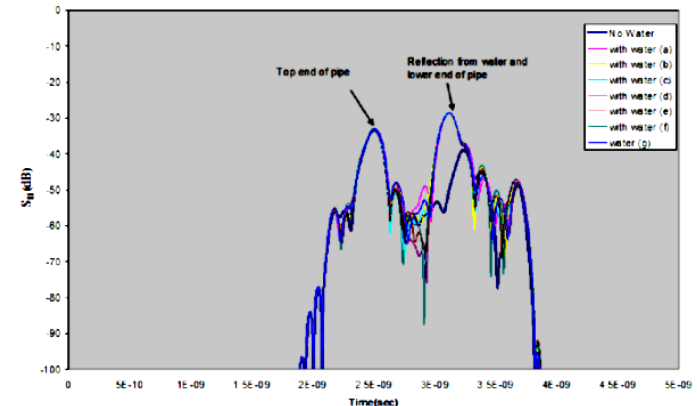

Fig. 20. Reflections from the surface of $21 \mathrm{~mm}$ flowing water inside a pipe

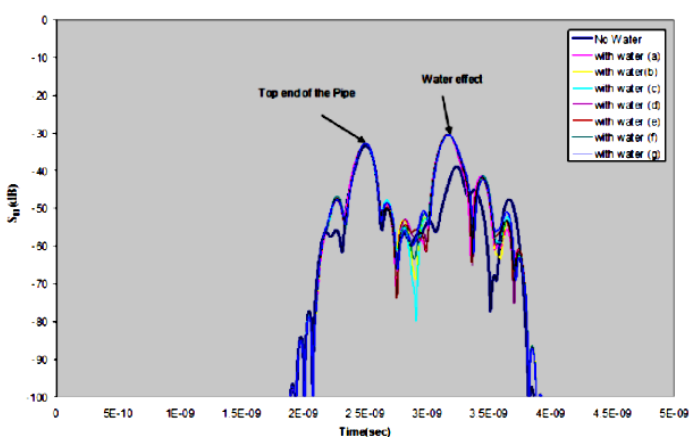

Fig. 21. Reflections from the surface of $9 \mathrm{~mm}$ flowing water inside a pipe

The next step was to transform these time domain results into distance domain, which was done and the results are shown in Figures 22 and 23. It has to be remembered that the pipe diameter "D" was approximately $110 \mathrm{~mm}$.

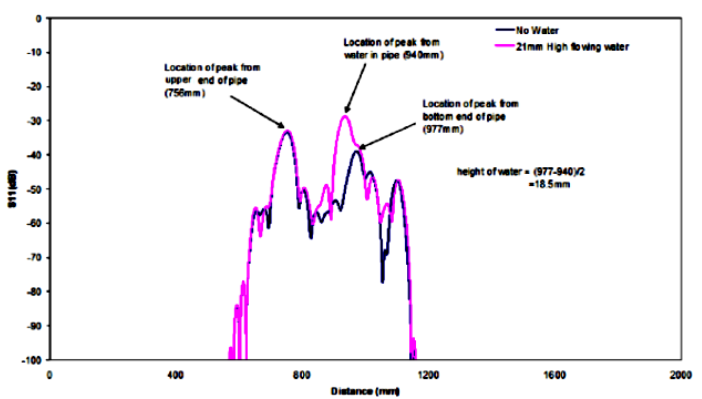

Fig. 22. Distance domain analysis of the results in time domain for $21 \mathrm{~mm}$ water flowing inside a pipe

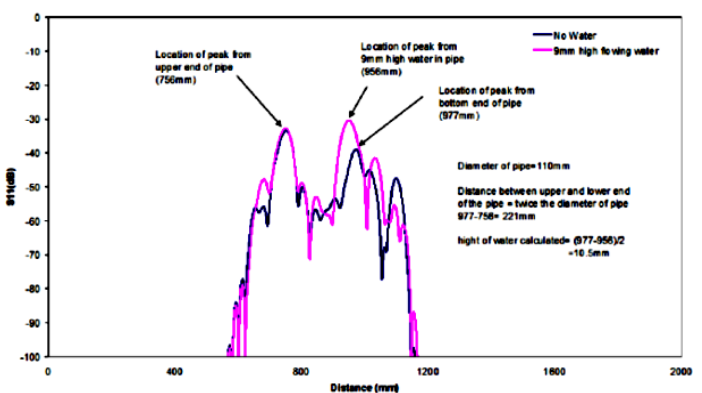

Fig. 23. Distance domain analysis of the results in time domain for $9 \mathrm{~mm}$ water flowing inside a pipe

\section{MATHEMATICAL ANALYSIS}

The location of the peak of the reflections from the upper end of the pipe, $d_{l}$ equals to $756 \mathrm{~mm}$. The location of the peak of the reflections from lower end of the pipe, $d_{2}$ equals to $977 \mathrm{~mm}$. The difference between these two should give a distance twice as big as the diameter of the pipe.

\section{$d_{1}-d_{2}=2 D=>977-756=221 \mathrm{~mm}$}

Which is nearly double the diameter of the pipe. Similarly the locations of the peaks of the reflections from the surfaces of the $9 \mathrm{~mm}$ and $21 \mathrm{~mm}$ high water inside the pipe were found to be at $10.5 \mathrm{~mm}$ and $18.5 \mathrm{~mm}$ respectively. 


\section{CONCLUSIONS}

This paper presented analysis, simulations, and measurements relating to the feasibility and principles of a device to measure water levels inside PVC piping using microwaves. Simulations showed that for a solution, measured results would need to be obtained during a time window between $2 \mathrm{~ns}$ and $10 \mathrm{~ns}$ dependent on the distance of the transducer from the target. Simulations showed that in the absence of noise and with a large bandwidth, water surface could be resolved to within a few $\mathrm{mm}$ with an accuracy of approximately $97 \%$. The top surface as well as the lower surface of the pipe could be detected.

The concept of gating was introduced here whereby the only period of time of interest is twice the time taken for the pulse energy to illuminate the surface of the water in the pipe. An idealized version of the system was modeled using a state of the art electromagnetic simulation package. The model consisted of a PVC sheet in front of a copper plate with water at varying levels and without water. The simulated results yielded some useful facts. The time of flight measurements could be easily translated into distance and the error was less than $4 \%$. The reflection form the water surface was approximately 12 times larger than the reflection from the pipe surface. The reflection form the pipe surface was unaffected by the level of water in the pipe. The main period of interest in the measurements lasted 4ns. The experimental measurements gave the following points as worth noting:

- All four water heights had distinct peaks.

- Reflections from below the floor caused many standing waves indicating that gating would be required for both pulse and phase type EMD systems.

- The period of interest is less than $2 \mathrm{nsec}$ which is consistent with the model.

- Even with a possible metalized floor it was difficult to determine a fixed point in time/space for the bottom of the water container.

Based upon the obtained results it is apparent that a device of the type described here is entirely feasible.

\section{REFERENCES}

[1] J. M. Rueger, Electronic Distance Measurement: An Introduction Vol. 4, Springer, Berlin-Heidelberg-New York, 1996

[2] E. C. Fear, X. Li, S. C. Hagness, M. A. Stuchly, "Confocal microwave imaging for breast cancer detection: Localization of tumors in three dimensions", IEEE Transactions on Biomedical Engineering, Vol. 49, No. 8, pp. 812-822, 2002

[3] E. Fear, M. Stuchly, "Microwave detection of breast cancer", IEEE Transactions on Microwave Theory and Techniques, Vol. 48, No. 11, pp. 1854-1863, 2000

[4] H. Zhang, S. Y. Tan, H. S. Tan, "A novel method for microwave breast cancer detection", 2008 Asia-Pacific Microwave Conference, Macau, China, December 16-20, 2008

[5] U. Weinstein, A. Bernstein, E. Cohen, Microwave Monitoring of Heart Function, U.S. Patent US 2011/O130800 A1, 2016

[6] S. S. Kim, S. T. Kim, Y. C. Yoon, K.S. Lee, "Magnetic, dielectric, and microwave absorbing properties of iron particles dispersed in rubber matrix in gigahertz frequencies", Journal of Applied Physics, Vol. 97, No. 10, pp. 10F905-1-10F905-3, 2005
[7] K. E. Haque, "Microwave energy for mineral treatment processes-a brief review", International Journal of Mineral Processing, Vol. 57, No. 1, pp. 1-24, 1999

[8] G. S. Woods, D. L. Maskell, M. V. Mahoney, "A high accuracy microwave ranging system for industrial applications", IEEE Transactions on Instrumentation and Measurement, Vol. 42, No. 4, pp. 812-816, 1993

[9] M. I. Khattak, M. Shafi, G. Ahmad, N. Ullah, M. Saleem, "Effects of the Span of the Calibrating Frequency Scan on Resolution of Microwave Electronic Distance Measurements", Technical Journal, Taxila, Pakistan Vol. 19 No. 4, pp. 30-33, 2014

[10] Q. Shi, O. Kanoun, "Wire Fault Diagnosis in the Frequency Domain by Impedance Spectroscopy", IEEE Transactions on Instrumentation and Measurement, Vol. 64, No. 8, pp. 2179-2187, 2015

[11] Q. Shi, U. Troltzsch, O. Kanoun, "Analysis of the parameters of a lossy coaxial cable for cable fault location", Eighth International MultiConference on Systems, Signals \& Devices, Sousse, Tunisia, March $212-25,2011$ 